\title{
Reduziert Acetazolamid die Dauer der Beatmung?
}

Pharmakodynamische Untersuchungen ließen vermuten, dass invasiv mechanisch beatmete Patienten mit COPD und metabolischer Alkalose von den respiratorisch stimulierenden Effekten einer Acetazolamid-Therapie profitieren. C. Faisy und Kollegen aus Paris haben die Hypothese aufgestellt, dass diese Behandlung die Beatmungsdauer kritisch kranker Patienten verkürzt. In ihrer Untersuchung konnten sie diese Annahme jedoch nicht bestätigen. JAMA 2016; 315: 480-488

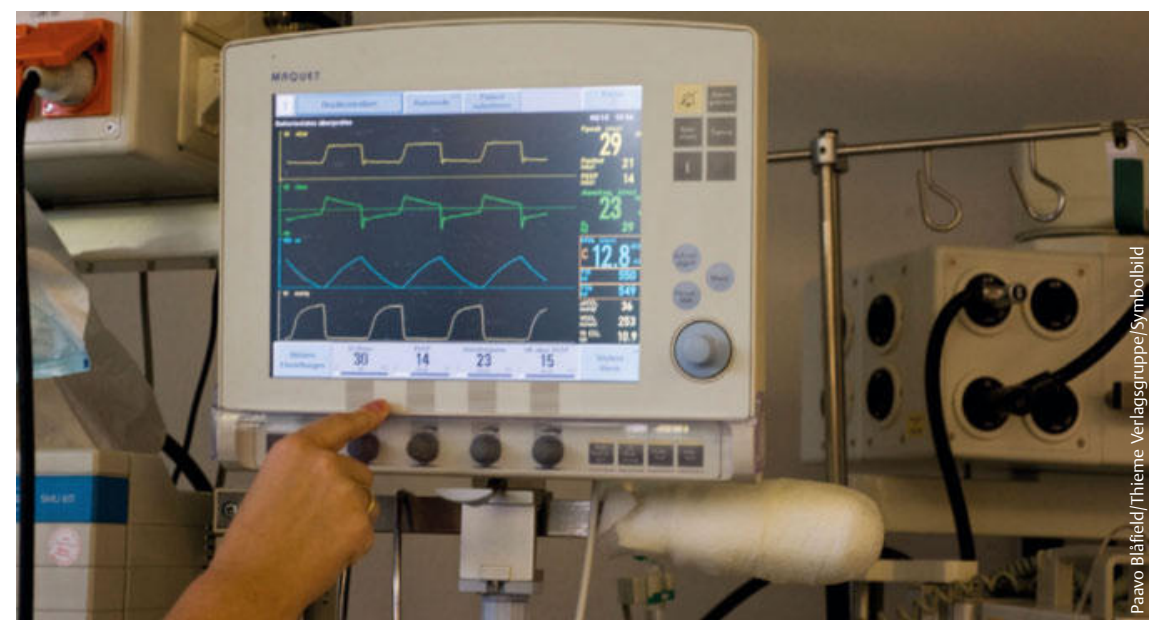

Die metabolische Alkalose ist eine der hauptsächlichen säurebedingten Beeinträchtigungen von Patienten mit COPD während der mechanischen Beatmung. Der Carboanhydrasehemmer Acetazolamid wirkt bei diesen Patienten als respiratorisches Stimulans. Er steigert das Atemminutenvolumen (AMV) über die Wirkung auf periphere und zentrale Chemorezeptoren. Mit den üblichen Dosen von 250-500 mg 1-mal oder 2-mal täglich ergaben sich in einigen Studien jedoch inkonsistente Wirkungen. In unkontrollierten Studien schienen die betroffenen Patienten wiederum von höheren Dosen zu profitieren.

\section{DIABOLO-Studie}

Die französischen Forscher haben daher untersucht, ob höhereDosenab $1000 \mathrm{mg} / \mathrm{d}$ die Dauer der mechanischen Beatmung bei kritisch kranken Patienten mit COPD und metabolischer Alkalose verkürzen können. Sie führten dazu in 15 Intensivpflegeeinheiten (IPE) die kontrollierte doppelblinde DIABOLO-Studie durch. In die Studie nahmen sie 382 von ursprünglich 694 gescreenten COPD-Patienten auf, die länger als 24 Stunden mechanisch beatmet werden sollten. Sie verteilten die Patienten randomisiert auf eine Gruppe, die sie mit 2-mal täglich 500-1000 mg Acetazolamid behandelten $(n=188)$ und eine Gruppe, die Placebo erhielt $(n=194)$. Sie verabreichten die Medikation intravenös bei Auftreten einer reinen oder gemischter Alkalose innerhalb von 48 Stunden nach der Aufnahme in eine IPE und führten die Behandlung während des Aufenthalts in der IPE für max. 28 Tage fort.

380 der rekrutierten Patienten nahmen an einer Intent-to-treat-Analyse teil. Das mittlere Alter betrug 69 Jahre, 272 waren
Männer (71,6\%). 379 Patienten (99,7\%) waren endotracheal intubiert. Das primäre Studienziel bestand in der Dauer der invasiven mechanischen Beatmung.

Sekundäre Studienziele waren:

- Veränderungen bei den arteriellen Blutgasen und respiratorischen Parametern,

- Dauer der Weaningphase (Beatmungsentwöhnung),

- Nebenwirkungen,

- Anwendung nicht-invasiver Beatmung nach der Extubation,

- Dauer des IPE-Aufenthalts und

- IPE-Mortalität.

\section{Keine signifikante Reduktion $\nabla$}

Die statistische Auswertung ergab keine signifikanten Unterschiede im Vergleich der Acetazolamid- mit der Placebogruppe hinsichtlich der mittleren Dauer der mechanischen Beatmung (136,5 vs. 163 Stunden; -16 Stunden; $p=0,17)$, Dauer der Beatmungsentwöhnung (18,7 vs. 22,9 Stunden; -0,9 Stunden; $p=0,36)$, der täglichen Veränderung des AMV (0,2 vs. 0,2 1/min; $\mathrm{p}=0,72$ ) oder des partiellen $\mathrm{CO}_{2}$-Drucks im arteriellen Blut (-0,5 vs. $-0,2 \mathrm{mmHg}$; Unterschied zwischen den Gruppen -0,2 mmHg; $\mathrm{p}=0,25)$. Andererseits nahmen die täglichen Veränderungen der Serumbikarbonat-Konzentration (Unterschied zwischen den Gruppen -0,8; p = 0,001) und die Anzahl der Tage mit metabolischer Alkalose (Unterschied zwischen den Gruppen -1; $\mathrm{p}=0,001$ ) unter Acetazolamid deutlicher ab als unter Placebo. Bei allen anderen sekundären Studienzielen gab es keine Unterschiede zwischen den Vergleichsgruppen. Das galt auch für die Anzahl schwerer Nebenwirkungen (6 unter Acetazolamid vs. 7 unter Placebo).

\section{Fazit}

Nach Angaben der Autoren führt die Behandlung mit hohen effektiven Dosen von Acetazolamid bei mechanisch beatmeten Patienten, die unter COPD und metabolischer Alkalose leiden, nicht zu einer statistisch signifikanten Reduktion der Dauer der künstlichen Beatmung. Dennoch sehen sie den Unterschied zwischen den Gruppen von 16 Stunden als klinisch bedeutsam an und halten es für möglich, dass die Studie eine zu geringe Teststärke/Stichprobengröße aufwies, um eine statistische Signifikanz zu erreichen.

\section{Dr. Volker Kriegeskorte, Buchloe}

